\title{
Wnt Signaling Regulates Intermediate Precursor Production in the Postnatal Dentate Gyrus by Regulating Cxcr4 Expression
}

\author{
Youngshik Choe $^{a} \quad$ Samuel J. Pleasure ${ }^{a-d}$

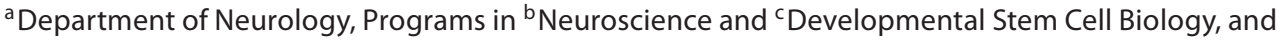 \\ dEli and Edythe Broad Center of Regeneration Medicine and Stem Cell Research, University of California, \\ San Francisco, Calif., USA
}

\section{Key Words}

Wnt signaling • Sonic hedgehog signaling • Dentate gyrus • Intermediate progenitor cell

\begin{abstract}
Previous studies have examined the role of diverse signaling pathways in dentate neurogenesis, but how these signaling pathways are integrated remains unknown. Using mice that allow genetic manipulation of type 1 radial progenitors in the dentate, we show that forced induction of Wnt signaling leads to expansion of the intermediate progenitor cell (IPC) pool while selective activation of Sonic hedgehog (Shh) signaling drives neurogenesis without significant expansion of IPCs. Thus, both Wnt and Shh signaling are proneurogenic, but they act in distinct manners when their signaling is forced in subgranular zone radial progenitors. We examined potential targets of the Wnt pathway in these cells and found that Cxcr4 is a direct target of Lef1 in dentate gyrus progenitors and that loss of Cxcr4 in postnatal neurogenesis decreases the production of IPCs. This suggests that Wnt activation of dentate gyrus progenitors induces $\mathrm{Cxcl} 12$ signaling by regulating receptor expression. This study provides evidence that distinct morphogenic pathways have notably different roles in regulating ongoing dentate neurogenesis.
\end{abstract}

Copyright $\odot 2012$ S. Karger AG, Basel

\section{KARGER}

Fax +41613061234

E-Mail karger@karger.ch

www.karger.com
(C) 2012 S. Karger AG, Basel

$0378-5866 / 12 / 0346-0502 \$ 38.00 / 0$

Accessible online at:

www.karger.com/dne

\section{Introduction}

Newborn neurons are produced in adult rodents in two distinct neurogenic niches - the subventricular zone and the subgranular zone (SGZ) in the dentate gyrus (DG). In the dentate, the adult neurogenic niche is formed by the interaction of multiple cell types including type 1 progenitors (radially oriented progenitors with glial markers), located at the border of the hilus and dentate granule cell layer. The position of type 1 progenitors in the SGZ allows them access to a range of neurogenic regulators, including Wnt [1] and Sonic hedgehog (Shh) [2], and both of these pathways are potent regulators of neurogenesis. Lineage tracing of Shh-responding cells previously showed that these are the postnatal quiescent neurogenic astrocytes which first appear in the dentate at late embryogenesis in the DG [3]. Shh signaling mutants show a loss of neurogenic stem cells as well as diminished cell proliferation during early postnatal dentate development [4-6]. Also, mice with Wnt pathway mutations have long been known to have severe developmental dentate phenotypes [1, 7-9], but manipulation of the Wnt pathway in adult rodents also leads to major defects in dentate neurogenesis [1], in part because of the role of Wnt in regulating key neurogenic factors like NeuroD in the neuroblast cells [8]. How these pathways are integrated by type 1 
stem cells in the SGZ and whether their effects are similar or redundant has not been addressed previously.

The most dramatic expansion of DG size as well as the number of DG granule neurons occurs during the first 2 weeks of postnatal life. During this time, there is extensive reorganization of the dentate stem cell niche [10-12]. However, by the end of this time, the dentate neurogenic niche is formed and the DG stem cells are localized specifically in the SGZ. Formation of the embryonic DG is regulated by Wnt from the cortical hem $[7,11,13]$, and the Shh signaling pathway begins to be prominent at the late embryonic-perinatal period $[3,6]$. There is also abundant evidence that Shh and Wnt signaling continue to be important in later ongoing dentate neurogenesis (after P15) $[1-3,5,14]$, but there is little understanding of how these pathways are integrated in their functions in the DG. We are particularly interested in the role of the Shh and Wnt signaling pathways in regulating the behavior of type 1 DG stem cells once the SGZ has been established and how the effects of these pathways differ and collaborate. Here, we examine how both Wnt and Shh signaling regulate the behavior of DG neural stem cells and show that Cxcr4 is an important downstream target of the Wnt pathway in the dentate neurogenic niche.

\section{Materials and Methods}

\section{Animals}

Gli1-CreERt2 [3], hGFAP-Cre [15], Cxcr4 $4^{\text {flx }}$ [16], Ctnnb1 ${ }^{(\text {Gof })}$ [17] and SmoM2 [18] mouse lines were previously reported. RosaYFP mice were obtained from Jackson Laboratory (Bar Harbor, Me., USA). Experimental mice were generated by crossing Creexpressing male mice with the homozygous conditional mutant female mice. All the lines were maintained in mixed genetic background. The day of vaginal plug was considered to be embryonic day 0.5 (E0.5) and the day of birth as postnatal day 1 (P1). Electroporation of green fluorescent protein (GFP) and CA-Cxcr4 (asparagine at 121 to alanine) was conducted using pregnant CD1 mice as described in the previous paper [19]. Mouse colonies were housed at the University of California, San Francisco (UCSF), in accordance with the National Institutes of Health and the UCSF guidelines.

\section{In situ Hybridization}

All experiments were done by comparing control and mutant sections stained on the same slides to reduce variation. Sections were prepared at $20-\mu \mathrm{m}$ thickness and stored at $-80^{\circ} \mathrm{C}$ until use. Slides were warmed to room temperature and treated with proteinase $\mathrm{K}(50 \mu \mathrm{g} / \mathrm{ml})$ for $10 \mathrm{~min}$ and fixed with $4 \%$ paraformaldehyde for $10 \mathrm{~min}$. Acetylation was performed using $0.25 \%$ acetic anhydride in $0.1 \mathrm{M}$ triethanolamine, $\mathrm{pH} 8.0$, for $10 \mathrm{~min}$, followed by three $1 \times$ PBS washes. Slides were incubated with hybridization buffer [ $50 \%$ formamide, $5 \times$ SSC, $0.3 \mathrm{mg} / \mathrm{ml}$ yeast tRNA, 100 $\mathrm{mg} / \mathrm{ml}$ heparin, $1 \times$ Denhardt's, $0.1 \%$ Tween 20, 0.1\% CHAPS (3-[(3-cholamidopropyl)dimethylammonio]-1-propanesulfonate), $5 \mathrm{~mm}$ EDTA] for $10 \mathrm{~min}$ at $65^{\circ} \mathrm{C}$, followed by overnight incubation with a digoxigenin (DIG)-labeled probe $(500 \mathrm{ng} / \mathrm{ml})$. Five high-stringency washes were performed with $0.2 \times$ SSC at $65^{\circ} \mathrm{C}$. Slides were then incubated with alkaline phosphatase-conjugated anti-DIG and nitroblue tetrazolium/5-bromo-4-chloroindolyl phosphate (Roche) for the detection of signals. Non-radioactive DIG-labeled probes were prepared using cDNA templates of Axin 2 and Cxcr4 which were obtained from polymerase chain reaction (PCR) amplification using primers designed according to the sequence information from Allen Institute for Brain Science.

\section{Tamoxifen Induction and Bromodeoxyuridine Labeling}

Tamoxifen (TM; Sigma) stock was prepared by dissolving the powder in corn oil (Sigma) at $20 \mathrm{mg} / \mathrm{ml}$. P15 mice were injected with a single intraperitoneal dose of $2 \mathrm{mg} / 10 \mathrm{~g}$ per animal except for the conditional inhibition of a Cxcr $4^{\text {flx }}$ allele that was accomplished with daily injections from P15 to P17. Bromodeoxyuridine (BrdU; Roche) dissolved in PBS (10 mg/ml) was injected intraperitoneally at a dose of $100 \mathrm{mg} / \mathrm{kg}$ per animal for the labeling of S-phase cells.

\section{Immunohistochemistry and Confocal Microscopy}

Mouse brains were collected after intracardiac perfusion with $4 \%$ paraformaldehyde in $1 \times$ PBS followed by cryoprotection with $30 \%$ sucrose/PBS overnight. Optimal cutting temperature-embedded tissues were processed in a cryostat at $30-\mu \mathrm{m}$ sections for immunostaining and at $20-\mu \mathrm{m}$ sections for in situ hybridization. Primary antibodies used for immunostaining were chicken antiGFP (1:1,000; Aves Labs), rabbit anti-GFP (1:1,000; Invitrogen), rabbit anti-Prox1 [20], rat anti-BrdU (1:200; Novus), mouse antiNeuN (1:100; Millipore), rat anti-Ctip2 (1:500; Abcam), rabbit anti- BLBP (1:500, Chemicon; 1:300, Abcam), rabbit anti-Ki67 (1:200; LabVision), rabbit anti-Tbr2 (1:200; Abcam), and rabbit anti-LEF1 (1:100; Cell Signaling Technology). To normalize cell counting, we used medioseptal regions of the DG at +400 to +800 $\mu \mathrm{m}$ from the appearance of the DG (set as $0 \mu \mathrm{m}$ ) and presented the number of cells per single DG. We excluded temporal regions of the DG since the GFP+ cells were too crowded to count. We selected 3-6 sections from different mice to stain a single marker. We counted all GFP-positive cells in the area of confocal images that were acquired at the Nikon Imaging Center at the UCSF using an upright Nikon $\mathrm{C} 1$ spectral confocal microscope equipped with 405-, 488- and 561-nm lasers with $10 \times, 20 \times$ and $63 \times$ objective.

\section{Chromatin Immunoprecipitation and Reporter Assay}

We used the ChIP-IT system (Active Motif) using rabbit and mouse anti-Lef1 antibody (Cell Signaling Technology and Abnova, respectively) for chromatin immunoprecipitation (IP) and used rabbit immunoglobulin $\mathrm{G}$ ( $\mathrm{IgG}$ ) as a negative control IP. Quantitative PCR was conducted using immunoprecipitates collected from three E17.5 embryonic DG tissues using the 7500 Fast Real-Time PCR system (Applied Biosystems). Primer pairs were Cxcr4p_F (cccaggaaactgcagtcatg) and Cxcr4p_R (cactccgttcgcagatgtac) for the promoter of mouse Cxcr4. Independent quantitative PCR was performed three times using a SYBR Green PCR 
master mix (Applied Biosystems), and the $\Delta \Delta$ Ct value was used to present fold differences of relative binding activities using a value obtained from rabbit IgG without primary antibodies as a reference.

For the luciferase reporter assay, we used the dual luciferase system (Promega) using pGL3-luc (Promega) containing an SV40 minimal promoter as a vector backbone and a $\mathrm{pRL}-\mathrm{TK}$ vector to normalize the transfection efficiencies. Using Lipofectamine 2000 (Invitrogen), we used a total of $2 \mu$ g plasmid DNA per single transfection in COS7 cells and collected cell extracts at $18 \mathrm{~h}$ after transfection. We cloned a $2.5-\mathrm{kb}$ proximal region of the mouse Cxcr4 promoters into the pGL3-luc vector. Deletion of the promoter was conducted by PCR using primer combination of sense primers, Cxcr4p-s0 (GGGCTACTCAGCTATTACTAAC), Cxcr4p-s1 (GGGCTACATCATGAAAGGTAG) and Cxcr4ps2m (gccaaatattatccttgaaag) and an antisense primer Cxcr4p-s2r (cacaggtctctttagctcctg) shown as a diagram in figure 4 .

\section{Statistical Analysis}

Values are presented as the mean \pm SEM in plots. For statistical analyses, we used Student's t test and one-way ANOVA, and the $p$ values of Tukey's post hoc test were presented after ANOVA (Graph Pad and Excel Software).

\section{Results}

\section{Gli1-CreERt2 Allows Manipulation of the Dentate}

\section{Neural Stem Cell Pool}

Our initial task was to validate a useful genetic tool for manipulating the DG stem cells selectively. One barrier has been the identification of adequate markers to identify the stem cells accurately. Previous studies have shown that they express Nestin, Sox2, BLBP and GFAP; however, each of these markers is also expressed by non-neurogenic astrocytes in the DG and elsewhere in the cortex. In order to identify a better marker, we examined the expression of Lef1 using an antibody suitable for immunohistochemisty. Lef1 is a required determinant of dentate granule cell specification [7], but expression has not been carefully examined in the dentate lineage. At P15 when the SGZ is established, Lef1 is expressed in cells sharing expression of the known radial progenitor marker BLBP, but not overlapping with either Tbr2 (an intermediate progenitor marker) or Proxl (a postmitotic dentate granule cell marker; fig. 1a). Quantification of this staining showed that essentially all of the Lef1+ cells expressed BLBP, while virtually none expressed Tbr2 or Prox1 (fig. 1c). Thus, Lef1 appears to be a useful marker for DG stem cells and may indicate that this population of cells is poised to respond to Wnt ligands.

Next, we took advantage of the Glil-CreERt2 mouse line that allows labeling of cells in the SGZ which are ac- tively responding to Shh signaling [3] and crossed these with Rosa-YFP reporter mice. The benefit of the Gli1CreERt2 line over Nestin-CreERt2 lines in our study is that we can label cells actively responding to Shh signaling at the time of TM injection. To take advantage of this line for examining the short-term labeling of Shh-responsive cells, we wanted to use only a single injection of TM at P15 followed by analysis of the labeled cells after 48 h. Glil-CreERt2 recombined cells (after TM injection at P15, then examined $48 \mathrm{~h}$ later) are present in the SGZ. The labeled cells were costained with Blbp, GFAP and Sox 2 which label SGZ radial glial (RG) stem cells, but not with Tbr2, for neurogenic transiently amplifying cells, PSA-NCAM and DCX for the immature neuroblast cells, and Prox 1 for the mature DG granule neurons (data not shown). In addition, short-term analysis of the GFP-labeled cells combined with Lef1 staining showed that there is extensive overlap between the GFP+ and Lef1expressing cells and that these cells included some portion of the actively dividing BrdU+ cells after a 1-hour BrdU pulse (fig. 1b). In addition, we found that essentially all the GFP+ cells expressed both BLBP and Lef1 at P17, 2 days after TM (fig. 1b-d). Thus, we conclude that Lef1 marks a population of SGZ progenitors which express RG stem cell markers that are actively Shh responsive, and the Gli1-CreERt2 line could prove useful for manipulating these cells.

To confirm that the GFP+ cells in the SGZ persist as long-lived DG stem cells, we observed Gli1-CreERt2 recombined cells at different time points. Several weeks after labeling, a few GFP+ cells were costained with Lef1 in the SGZ, and most of the GFP+ cells had migrated into the dentate granule cell layer, and by P120, there were numerous cells in the granule cell layer coexpressing GFP and Prox1 (data not shown). Thus, the Gli1-CreERt2 is an exceedingly useful tool, allowing recombination in a cohort of Shh-responding DG stem cells with a single injection of TM at P15 and marking these cells and their progeny long term. We also attempted to use the Gli1-CreERt2 mice to mark DG stem cells with TM injection at adult stages, but found that a single injection was inadequate to consistently drive recombination in a sufficient cohort of cells for clear analysis. Because multiple TM injections over several days were required in the adult mice, possibly because the adult dentate stem cells are more quiescent than the pubertal dentate stem cells, it precluded the type of shorter-term analysis we are interested in, and we confined our analysis to mice after P15 single injection. 
Fig. 1. Specific expression of Lef1 in the DG stem cells. a Immunohistochemical staining for Lef1 colabeled with Blbp (glial stem cells), Tbr2 (transit amplifying cells) and Prox1 (granule neurons) in the DG at P15. b Colocalization of Lef1 and GFP reporter driven by Gli1-CreERt2. The upper panel shows the DG stained with Lef1 and GFP reporter recombined by Gli1CreERt2 with a single TM injection at P15 analyzed at P17. Higher-magnification images of the boxed area are shown in the lower panel. Immunochemical analysis of the SGZ was performed using anti-GFP with BrdU (1 h) and Lef1. DAPI is used as counterstain (blue). Scale bars $=200 \mu \mathrm{m}$. c, d Percentages of Lef1-positive cells costained with each marker (c) and of Lef1-positive cells with cell type markers and BrdU $(1 \mathrm{~h})$ at P17 (d) are presented. From 6 mice, 200 cells were counted for each marker $(n=6)$. Error bars indicate \pm SEM. Statistical comparisons were performed by Student's t test. ${ }^{* *} \mathrm{p}<0.001$.

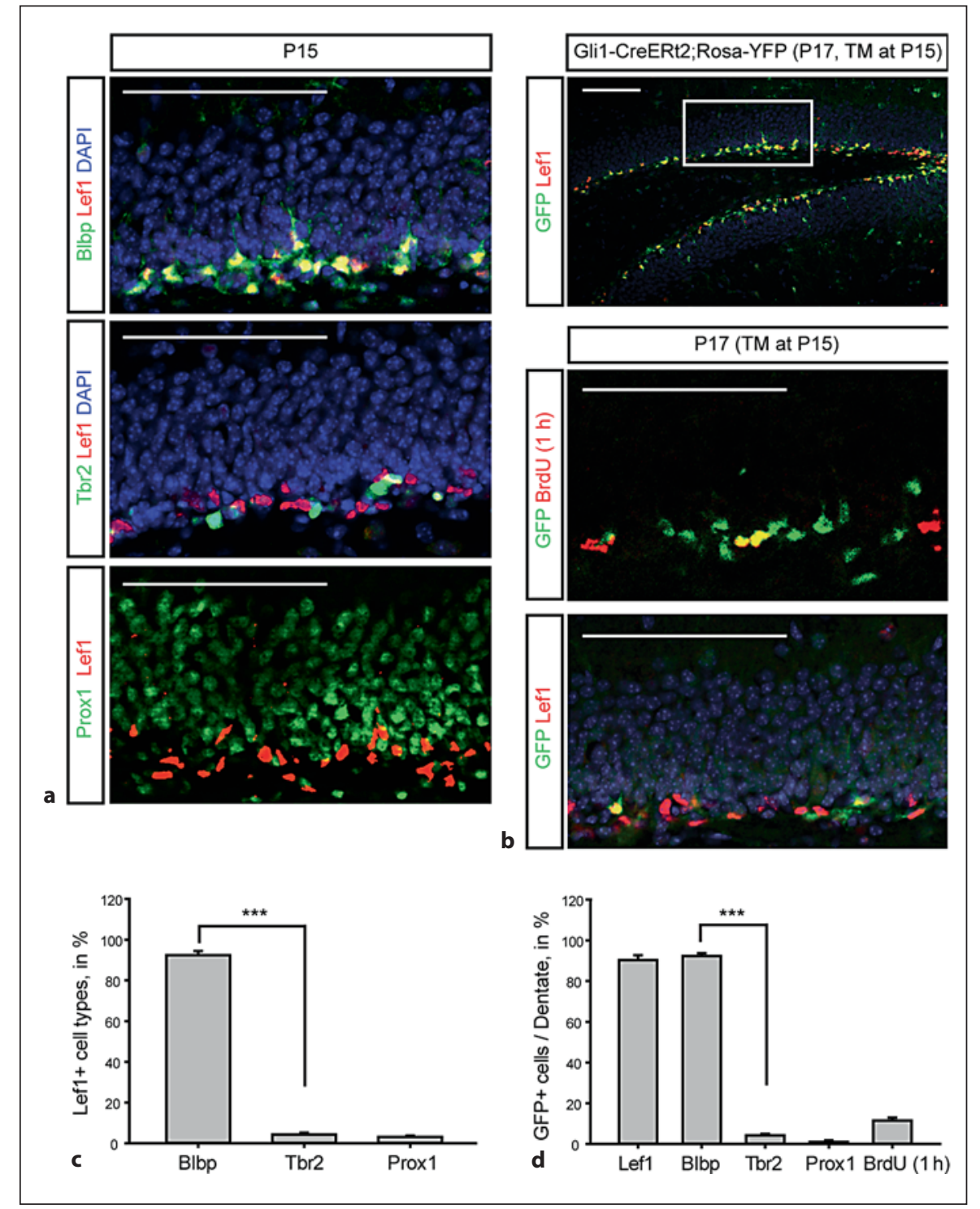

Distinct Neurogenic Consequences of Tonic $\beta$-Catenin and Hedgehog Signaling Activation in Dentate Stem Cells

Previous studies have demonstrated that both Wnt and Shh signaling are key regulators of postnatal dentate neurogenesis, but there has been little consideration of the different consequences of activation of these pathways. We reasoned that we could use the Gli1-CreERt2 mice to cell autonomously manipulate signaling in both of these pathways in a small cohort of homogeneous, actively Shh-responding RG stem cells. In this way, we would determine the lineage consequences of continued Shh signaling versus sustained Wnt signaling. To do this, we crossed Gli1-CreERt 2 with the floxed exon $3 \beta$-catenin allele $\left[\mathrm{Ctnnb} 1^{(\mathrm{Gof})}\right.$, that leads to stabilization and tonic activation of $\beta$-catenin] [17] and with the floxed SmoM2 allele (that allows expression of a constitutively active Shh signaling receptor) [18]. In each case, they will express dominantly active versions of these critical signaling proteins after Cre-mediated excision. We also crossed these mice with the Rosa-YFP reporter and used a single injection of TM at P15 to allow manipulation of a cohort of RG stem cells and then quantified the relative percentages of $\mathrm{GFP}+/ \mathrm{BLBP}+\mathrm{RG}$ stem cells, GFP+/Tbr2+ intermediate progenitors and GFP+/Prox1+ dentate granule cells in each condition after a 14-day survival period. Although the SmoM2 allele produces a SmoM2-GFP fusion protein that inserts into the cell membrane, it was undetectable, 
so we also made use of Rosa-YFP in these mice for the strong cytoplasmic GFP signal.

We found that both the SmoM2 and Ctnnb1 ${ }^{(\text {Gof })}$ mice had a substantial increase in the number of total GFP+ cells compared to controls, indicating an increase in cell production from RG stem cells driven by either tonic Shh or Wnt signaling at P30 (fig. 2a). In both cases, this corresponds to a large increase in the production of total neurons and a significant increase in the percentages of RG stem cells in the dentate from among the GFP-labeled cells. However, there was a major divergence in the two experimental conditions with regard to the fraction of intermediate progenitor cells (IPCs) produced (fig. 2b, c). In the tonic Wnt activation mice, there was a dramatic increase in the fraction of IPCs, while in the SmoM2 mice, there was a decrease in this fraction, implying that Wnt activation and Shh activation may have distinct effects on the mode of neurogenesis.

To determine the longer-term consequences of our hedgehog and Wnt signaling manipulations on dentate neurogenesis, we wanted to allow mice to survive for longer times after single injection at P15. One complication we found was that survival was reduced in the Gli1CreERt2;Ctnnb1 ${ }^{(\mathrm{Gof})}$ mice starting about 20 days after TM injection. This appeared to be due to a defect in subepithelial mesenchymal cells that have excess cell death leading to skin defects (data not shown). Thus, to allow Gli1-CreERt2; Ctnnb1 ${ }^{\text {(Gof) }}$ mice to survive, we had to decrease the dose of TM to half, making direct comparison of cell numbers between conditions impossible. However, we were able to compare the percentages at P60 (45 days after TM treatment) as we did at earlier survival times and found that again the activation of Wnt signaling leads

Fig. 2. Distinct lineage effects of $\beta$-catenin/Wnt and hedgehog signaling activation in dentate stem cells. Gli1-CreERt 2 mice were crossed with SmoM2 or Ctnnbl ${ }^{(\mathrm{Gof})}$ conditional mice with RosaYFP reporter (we used GFP for the label of YFP). TM was given at P15 and mice were analyzed at P30 (a-c) and at P60 (d, e). Scale bars $=200 \mu \mathrm{m}$. Error bars indicate \pm SEM. Statistical comparisons were performed by Student's t test or ANOVA followed by Tukey's multiple comparison post hoc test. The $\mathrm{p}$ values from Tukey's post hoc comparisons are presented after ANOVA $(n=6)$. ${ }^{*} \mathrm{p}<0.05 ;{ }^{* * *} \mathrm{p}<0.001$. NS = Not significant. Twenty-six (GFP, P30), 30 (SmoM2, P30), 30 [Ctnnb1 $\left.^{(\text {Gof })}\right], 20$ (GFP, P60), 18 (SmoM2, P60), 20 (GFP, $1 \mathrm{mg} /$ animal, P60) and 20 [Ctnnbl $^{\text {(Gof) }}$ sections were used for the measurement, and all GFP-positive cells in the confocal images were used for counting. a Total Gli1CreERt 2 recombined GFP-positive cells are presented. Upper and lower blades of the DG did not show significant differences. Schematic drawings represent conditional activation of target genes by to dramatic and significant increases in the percentage of IPCs in the cell cohort compared to Shh signaling activation or controls (fig. $2 \mathrm{~d}, \mathrm{e}$ ). The total number of cells labeled with GFP and cell type-specific markers clearly demonstrate that the Tbr2 population is also increased by Wnt signaling at this age (fig. $2 \mathrm{f}$ ).

However, tonic $\beta$-catenin activation does seem to hinder the generation or survival of postmitotic Prox1positive granule neurons compared to Shh signaling activation. This leads to the somewhat paradoxical finding that $\mathrm{Ctnnbl}^{(\mathrm{Gof})}$ mice showed slightly decreased dentate granule cell layer areas (ANOVA, $\mathrm{p}<0.05$ ) compared to the GFP or SmoM2 mice (online suppl. fig. 1; for all online supplementary material, see www. karger.com/doi/10.1159/000345353). This implies that neurons with tonic expression of Ctnnb1 ${ }^{(\mathrm{Gof})}$ may have difficulty integrating into the DG and are likely lost by cell death over time. We did not see any clear increase in cell death in the Ctnnb1 ${ }^{\text {(Gof) }}$ mice (data not shown), but we suspect this is due to the difficulty in choosing a specific time point for examination during the longer survival after TM treatment. Over this length of time, even a very small and imperceptible increase in cell death would be significant and lead to changes in overall neuronal survival but would be near impossible to document. However, we did see evidence that the neurons in Ctnnb1 ${ }^{(\mathrm{Gof})}$ mice do have immature dendritic trees, likely implying an inability to integrate into the dentate either as a primary or secondary effect of the Ctnnbl $1^{(\mathrm{Gof})}$ expression (online suppl. fig. 2). This is probably due to direct roles of $\beta$-catenin in dendrite development that are not necessarily dependent on Wnt signaling [21, 22]. To assess this, we measured the average number of dendritic

CRE recombinases. b Immunohistochemical analysis of the DG at P30 with GFP and cell type markers. c Percentage of cell types resulting from recombination by Gli1-CreERt2 using RosaGFP control, SmoM2 and Ctnnb1 ${ }^{\text {(Gof) }}$ conditional alleles. Figures are labeled with genotypes of conditional alleles without Gli1CreERt2. d Immunohistochemical analysis of the DG at P60 with GFP and cell type markers. e Percentage of cell types resulting from recombination with Glil-CreERt2 using Rosa-YFP control, SmoM2 and Ctnnb1 ${ }^{(\mathrm{Gof})}$ conditional mice. For Ctnnb1 ${ }^{(\mathrm{Gof})}$, we used TM at $1 \mathrm{mg}$ /animal to increase animal survival and compared them with same-dose-injected control animals. f Stacked bar graphs show the total number of double-positive cells (GFP with markers) to reveal the increased Tbr2-positive population by $\beta$-catenin activation. Statistical analysis was conducted between the same makers of GFP mice versus SmoM2 or Ctnnbl ${ }^{(\mathrm{Gof})}$ mice, i.e. Tbr2 of GFP mice versus Tbr2 of SmoM 2 mice, or Tbr2 of GFP mice versus Tbr2 of Ctnnb1 mice. 

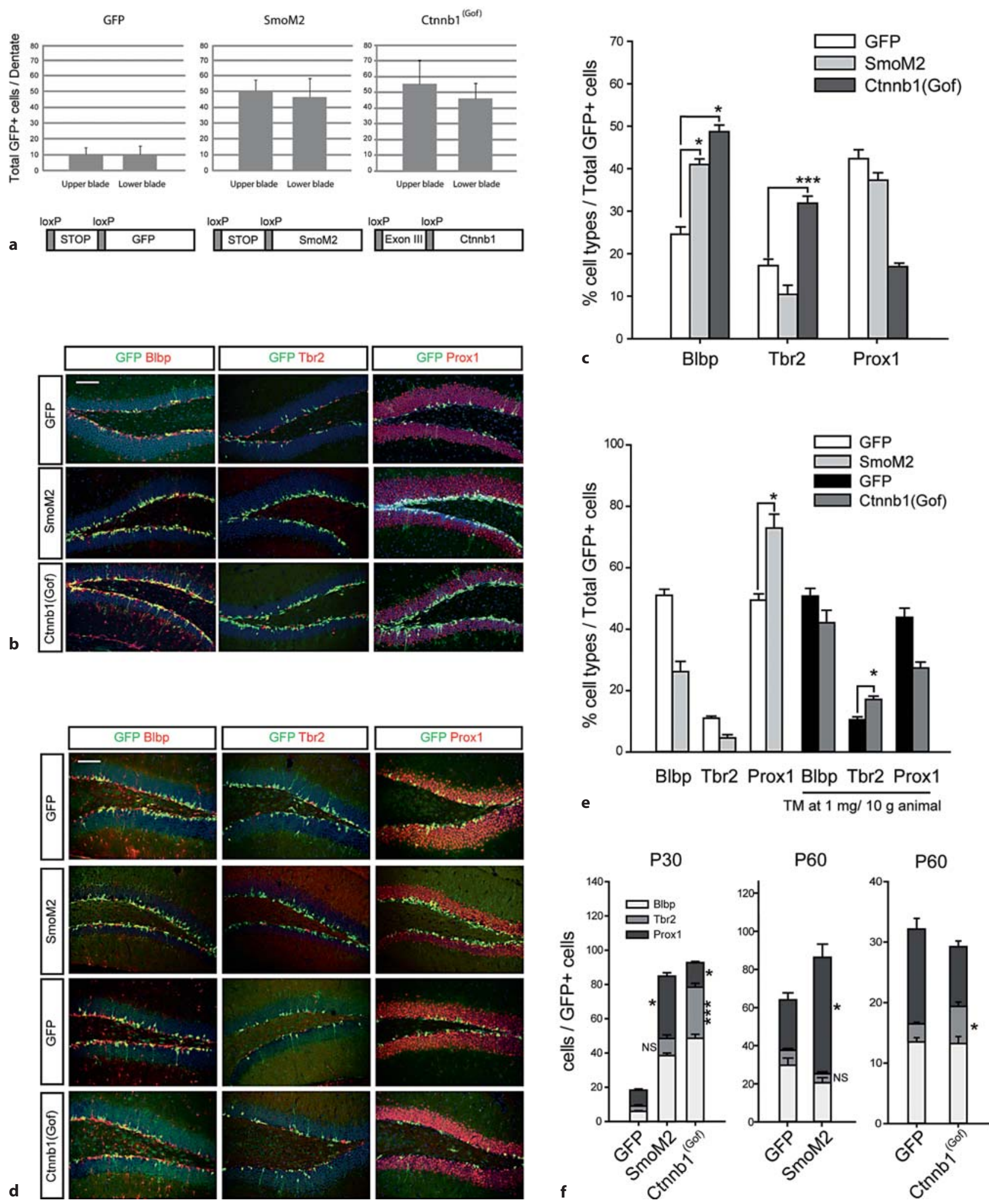
branches labeled with GFP from 75 Prox1+ granule neurons and found significantly simplified neuronal dendritic morphologies in Ctnnb1 $1^{\text {(Gof) }}$ mice at P60 (online suppl. fig. 2). This indicates that the expression of stabilized $\beta$-catenin during maturation of granule neurons hinders dendritic elaboration and likely contributes to the difficulty for these new neurons to integrate into the dentate circuitry.

\section{Cxcr4 Is a Target of the Wnt Pathway in the RG Stem}

Cells That Regulates the Production of Dentate IPCs

Our data suggest that activation of the $\beta$-catenin signaling pathway supports the production of cells with IPC markers in the dentate neurogenic lineage. To be sure that this phenotype was associated with expansion of the rapidly dividing pool of progenitors, we examined whether Wnt signaling activation increases the numbers of BrdUlabeled cells. We introduced TM at P15 and injected BrdU $(30 \mathrm{mg} / \mathrm{kg}$ ) four times at 6-hour intervals on P19 to saturate the proliferating cells. The number of BrdU/GFP double-positive cells was 2.5 times higher in the Gli1CreERt2;Ctnnb1 ${ }^{\text {(gof) }}$ mutants, confirming that the IPC pool is expanded (fig. 3a, b). Gli1-CreERt2;SmoM2 mice also showed significant increase in cell proliferation; however, the clusters of GFP+;BrdU+ cells were more prominent in the Gli1-CreERt2;Ctnnb1 mice, probably indicative of intermediate progenitor expansion [23].

Thus, we suspect that transcriptional targets of the Wnt pathway in these cells are likely to be important in the production of IPCs. First, to demonstrate that tonic activation of the Wnt pathway leads to direct transcrip- tional activation of Wnt targets in the SGZ, we examined the expression of a well-described Wnt transcriptional target - Axin2 [24]. We compared the expression of Axin2 in the SGZ in GFP controls to mice expressing SmoM2 or Ctnnb1 ${ }^{\text {(Gof) }}$ at 5 and 15 days after Gli1-CreERt 2 activation with a single dose of TM. It is clear that in these mice, Axin2 expression is dramatically increased in the SGZ of mice expressing stabilized $\beta$-catenin compared to the other two conditions (fig. $3 c$, d). NeuroD transcription factors are known to promote neurogenesis by activation of Wnt signaling in the dentate neuroblasts [8]; however, we did not see the increased expression of NeuroD1 in the P40 Gli1-CreERt2;Ctnnbl ${ }^{\text {(gof) }}$ mutant mice (data not shown). Thus, tonic activation of Wnt signaling in the type 1 DG stem cells seems to induce IPCs through other targets. We have previously noted that the distribution of Wnt transcriptional signaling in the developing medial cortex is quite similar to the expression of $\mathrm{Cxcr} 4$, a receptor for Cxcl12 and a known regulator of dentate development [25], so we wondered whether Cxcr4 expression was also upregulated in SGZ cells after activation of Wnt signaling. Agreeing with previous reports [25, 26], Cxcr4 expression is prominent in the SGZ (fig. $3 \mathrm{c}, \mathrm{g}$ ) and is dramatically upregulated in mice that have had a recombination of the Ctnnb1 ${ }^{\text {(Gof) }}$ allele when compared to control or SmoM2 mice (fig. 3c, d). Along with the strong intensity of Cxcr4 expression in the Gli1-CreERt2;Ctnnb1 ${ }^{(\mathrm{Gof})}$ mice, the mice had increased numbers of expressing cells at P30 (fig. 3g). To determine whether Cxcr4 might be directly regulated by Wnt signaling, we examined the putative promoter region of the Cxcr4 gene and found three con-
Fig. 3. Lef1-driven activation of Cxcr4 gene expression in the dentate stem cells. Scale bars $=200 \mu \mathrm{m} .{ }^{*} \mathrm{p}<0.05 ;{ }^{* *} \mathrm{p}<0.005$; ${ }^{* * *} \mathrm{p}<0.001$. NS $=$ Not significant. $\mathbf{a}, \mathbf{b}$ Proliferation is driven by $\beta$-catenin/Wnt and SmoM2/Shh signaling activation. TM was injected at P15 followed by four BrdU injections at P19 at 6-hour intervals and the DG was stained with GFP and BrdU. The representative images of GFP and BrdU staining of Gli1CreERt2;Rosa-YFP, Gli1CreERt2;SmoM2 and Gli1CreERt2; Ctnnb1 ${ }^{(\mathrm{Gof})}$ mice (a) and a graph showing the increase in cell proliferation in Gli1CreERT2; Ctnnb1 ${ }^{(\mathrm{Gof})}$ mice (b) are presented. The asterisk indicates a cluster of BrdU-labeled proliferating cells ( $\mathrm{n}=6$; $^{* *} \mathrm{p}<0.005$, ANOVA followed by Tukey's post hoc test). $\mathbf{c}-\mathbf{g}$ Representative images of in situ hybridization of Axin2 and Cxcr4 using animals at P20 (c) and P30 (d) with a graph showing Cxcr4-positive cell numbers in the SGZ at P30 (g) are presented $(\mathrm{n}=3 ; 10$ sections from in situ hybridization were used for the measurement; ${ }^{*} \mathrm{p}<0.05$, ANOVA followed by Tukey's post hoc test). e Chromatin IP of the mouse Cxcr4 promoter with Lef1 antibody. The representative gel image of chromatin IP is presented. The relative level of binding activity is calculated by the $\Delta \Delta \mathrm{Ct}$ values obtained by normalizing values using rabbit IgG as a control ( 3 independent experiments were conducted). f Luciferase reporter assays using mouse Cxcr4 promoter are presented. The inset is a schematic representation of the binding sites (circles) or a mutated binding site (circle with X) of TCF/ LEF transcription factors on the $2.5-\mathrm{kb}$ promoter (straight lines) of the mouse Cxcr4 and the partial fragments of the Cxcr4 promoter containing TCF/LEF elements (angled lines). The luciferase value was normalized to a sample transfected with a pCAGEGFP (CTL, a backbone vector of Lef1 contructs) with each promoter construct $(n=4)$. $\mathbf{h}$, $\mathbf{i}$ GFP and CA-Cxcr4 expression constructs were electroporated into the E13.5 embryonic cortices to target RG cells and analyzed at E15.5. A representative image (h) and a graph (i) showing the number of Tbr2-positive cells stained with GFP in the subventricular zone are presented (from 3 independent experiments). Statistical comparisons were performed by Student's $t$ test between the control group and the Lef1-transfected group $(\mathrm{n}=4)$. 


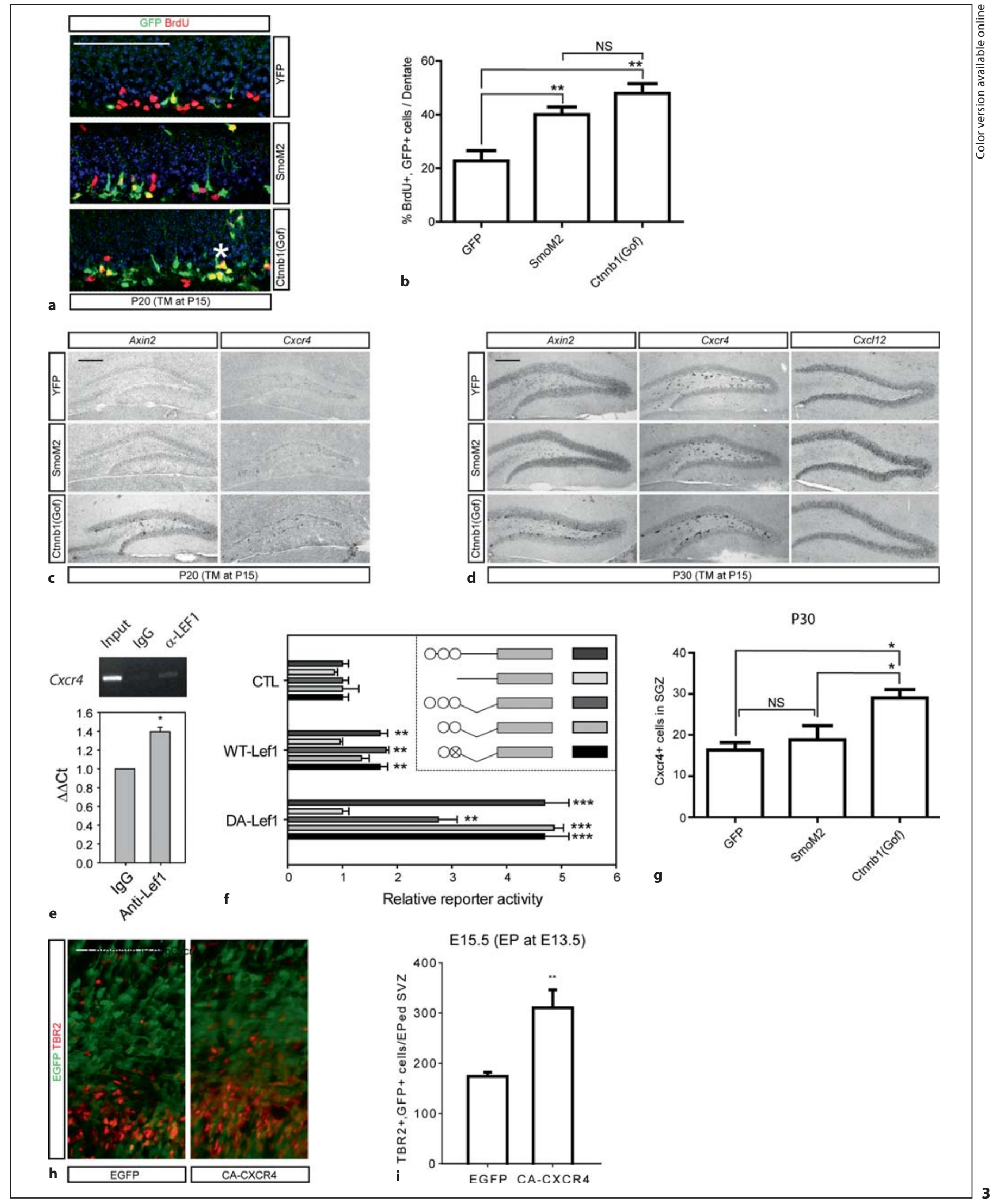


sensus Tcf/Lef-binding sites. Chromatin IP of complexes from brain extracts also showed that anti-Lef1 antibodies pull down fragments from the $\mathrm{Cxcr} 4$ promoter (fig. 3e). To more directly address the role of Lef1 in regulating Cxcr4 expression, we generated luciferase reporter constructs with the Cxcr4 promoter and compared their activity to control plasmids when cotransfected with Lef1. Indeed, Lef1 drives a moderate enhancement of Cxcr4 promoter activity, which is lost in deletion constructs lacking the three putative Lef1-binding sites (fig. 3f). Importantly, when we instead cotransfected a dominant active Lef1 construct with the DNA binding domain fused to the VP16 activation domain [27], we found much higher levels of induction of promoter activity that were again absent when the Lef1 binding sites were deleted (fig. 3f). Considering the specific expression of Lef1 in the RG cells and not in the IPCs, the induction of $\beta$-catenin expression should form heterodimeric activators with Lef1 in the RG cells and lead to downstream target activation and generation of IPCs from the RG cells. To determine whether ectopic signaling by Cxcr4 is able to induce IPC production from RG cells on its own, we electroporated a constitutively active form of Cxcr4 (CA-Cxcr4) into the embryonic ventricular zone and examined the production of IPCs compared to controls electroporated with GFP. We found that CA-Cxcr4 significantly expands the production of IPCs within the electroporated zone (fig. 3h, i). Interestingly a recent paper showed seemingly opposite results with stabilized $\beta$-catenin expression in the neocortex, where IPC production was decreased [28]. We believe that this distinction is probably due to specific induction of IPCs by CA-Cxcr4 and that $\beta$-catenin expression alone did not activate $\mathrm{Cxcr} 4$ signaling in the RG cells. This likely indicates important distinctions between the developmental potential of neural stem cells in the medial cortical anlage (where the dentate forms) and the neo-

Fig. 4. Neurogenesis defects in the DG of Cxcr4 conditional mutants. Statistical comparisons were performed by Student's t test or ANOVA followed by Tukey's multiple comparison post hoc test. The $\mathrm{p}$ values from Tukey's post hoc comparisons are presented after ANOVA. ${ }^{* *} \mathrm{p}<0.005 ;{ }^{* *} \mathrm{p}<0.001 . \mathrm{n}=6$ (minimum 20 sections were used per each counting). Scale bars $=100 \mu \mathrm{m}$. a The DG obtained from the Cxcr4 $4^{\text {flx }}$ conditional mice crossed with hGFAP-Cre at P15 were analyzed by immunohistochemistry with Tbr2 (transit amplifying cells), Blbp, Lef1 (dentate stem cells) and Ki67 (proliferating cells). b Representative images of P30 and P60 stained with neuronal markers (Ctip2, NeuN), transit amplifying cells (Tbr2) and proliferating cells (Ki67) are presented. cortex. It is quite likely in fact that the selective expression of Lef1 in the medial cortex is part of this distinction.

\section{Deletion of Cxcr4 Leads to Loss of Postnatal Dentate IPCs}

Previous studies from our lab showed that embryonic excision of Cxcr4 in the dentate leads to significant developmental defects and the loss of dividing progenitors from the embryonic and early postnatal dentate [29]. In that previous study, we found that after excision with Emx1Cre, the Cxcr4 $4^{\text {flx/flx }}$ mice show significant phenotypic rescue by P7, so that a phenotype was not visible at later postnatal stages [29]. At that time, we wondered if this was molecular compensation by another pathway; however, because of our new data, we decided to revisit this issue. We generated hGFAP-Cre;Cxcr $4^{\mathrm{flx} / \mathrm{fl} \mathrm{x}}$ mice and found that with this Cre driver, which has continued expression in the dentate stem cell lineage from embryonic through postnatal stages, we now uncovered a dramatic postnatal dentate phenotype (fig. $4 a, b$ ). In these mice, the DG is smaller than normal and has dramatically reduced numbers of Tbr2+ and Ki67+ IPCs in the SGZ at postnatal stages (fig. $4 \mathrm{a}, \mathrm{b}$ ). One obvious issue with these results is that the prominent embryonic phenotype in Cxcr4 mutant mice is probably responsible for significant consequences on the postnatal structure and function of the SGZ [29]. In the hGFAP-Cre;Cxcr4 $4^{\mathrm{flx} / \mathrm{fl} x}$ mice, this is apparent when we examine markers of the SGZ and the overall dentate structure - it is clear that the RG scaffolding and overall numbers of dentate stem cells are severely disrupted in these mice (fig. 4a). Therefore, to assess the role of Cxcr4 in a comparable situation with our Wnt and Shh manipulations, we generated Gli1CreERt2; $\mathrm{Cxcr} 4^{\mathrm{fl} x / \mathrm{flx}}$ conditional mutant mice after a daily injection of TM at P15 to P17. Since we injected TM three times, we obtained more GFP+ cells than in single injected

c, d Number of Tbr2-positive cells at P15 (c) and of Tbr2-positive cells at P30 (d). Het $=$ hGFAP-Cre $; \mathrm{Cxcr}^{\mathrm{flx} /+} ;$ Mut $=$ hGFAPCre; $\mathrm{Cxcr} 4^{\text {flx/flx }}$. e Double staining of GFP and Tbr2 from Gli1CreERt2;Rosa-YFP, Gli1-CreERt2;Ctnnb1 ${ }^{\text {(Gof) }}$;Rosa-YFP and Gli1-CreERt2;Cxcr4 ${ }^{\text {flx } / f l x} ;$ Rosa-YFP mice. TM was injected from P15 to P17 to achieve conditional mutation of the Cxcr4 $4^{\text {flx }}$ allele. f Double staining of GFP and Blbp using the DG obtained from Gli1-CreERt2;Rosa-YFP, Gli1-CreERt2;Ctnnb1 ${ }^{\text {(Gof) }}$;Rosa-YFP and Gli1-CreERt2;Cxcr4 ${ }^{\text {flx/flx }} ;$ Rosa-YFP. g, h Plots representing the number of cells costained with GFP and Tbr2 in the DG at P30 (g) and the number of cells costained with GFP and Blbp in the DG at P30 (h). 

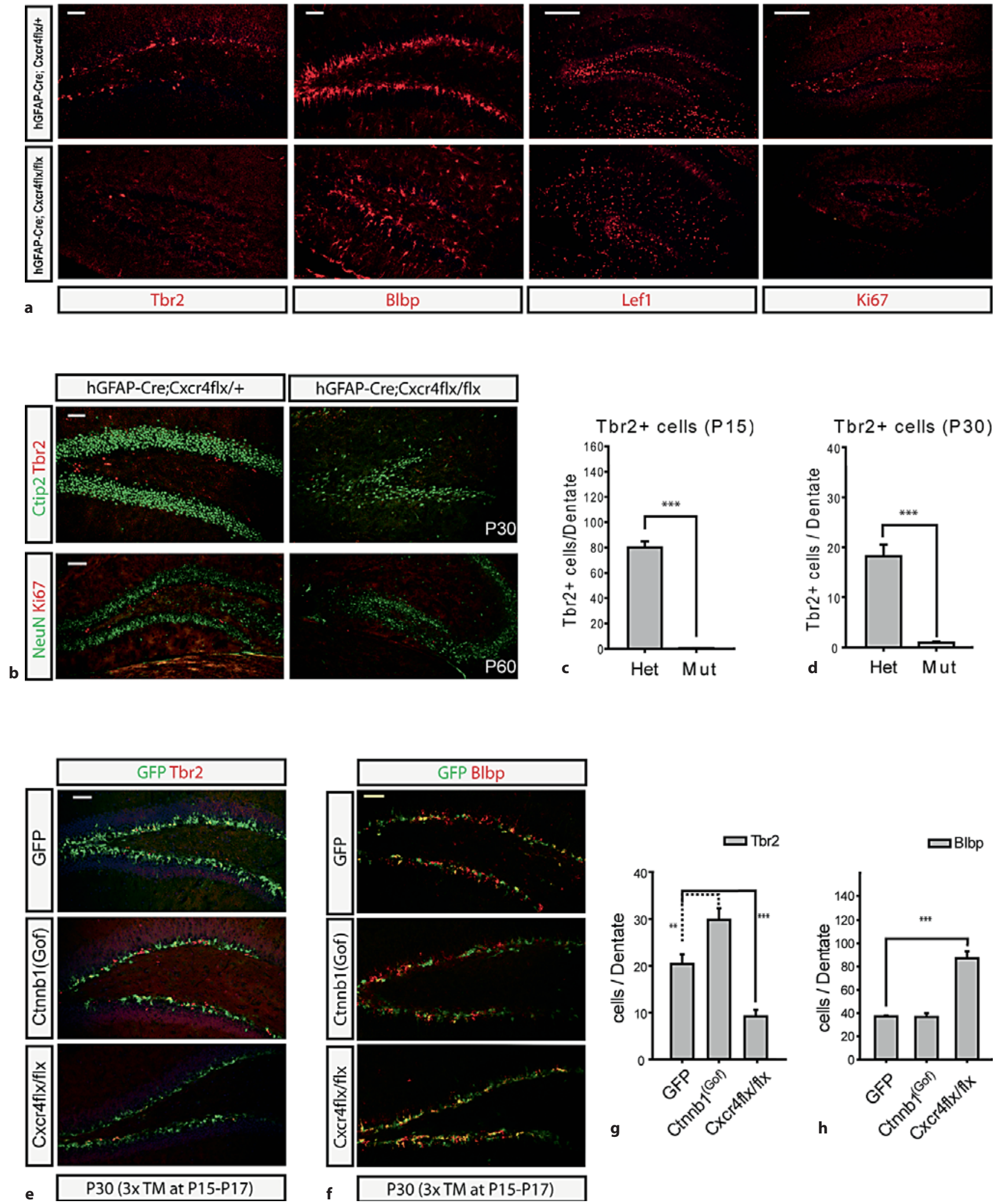
mice, which made it difficult to compare total GFP+ cells. Instead, we counted Tbr2+ IPCs that were also GFP+ in controls versus experimental littermates and found a significant decrease in the numbers of GFP+ IPCs in the mutants (fig. 4e, g). Twice more GFP+ cells were Blbp+ which implies gliogenic proliferation rather than neurogenic, IPC production in the absence of Cxcr4 expression in the mutant (fig. 4f, h). To rule out that the loss of IPCs was due to increased selective cell death of IPCs without Cxcr4, we examined cell death in these mice using staining for activated caspase- 3 and found no increase (online suppl. fig. 3). Thus, Cxcr4 is required in the dentate lineage for efficient production of IPCs in the postnatal dentate.

\section{Discussion}

Recent studies have shown that neurogenesis in the dentate is quite plastic, allowing environmental inputs to modulate the cellular composition of the dentate. Environmental influences such as stress, psychiatric disease, cognitive enrichment and exercise all somehow interact with the variety of signaling pathways that control maintenance and neurogenesis in dentate neural stem cells to shape the pool of new neurons in the dentate [30]. We found a quite enhanced rate of neurogenesis at P15 compared to adult stages, which allowed us to use the Gli1CreERt 2 mice to cell autonomously manipulate target alleles in a small cohort of DG stem cells after a single TM injection. Conditional labeling of DG stem cells with a single TM injection allowed more refined manipulation of stem cells in the SGZ than in previous studies using other Cre lines that also have varying degrees of activity in rapidly dividing precursors in the dentate [31-33]. That the short-term labeled Gli-CreERt 2 cells (using a YFP reporter, stained with GFP antibodies) also expressed Lef1 suggested that these cells are poised to respond to Wnt available in the SGZ niche. Since these cells are also identified by their strong Shh responsiveness (because of their Gli1 expression), we thought this was an interesting opportunity to test the consequences of manipulating signaling through these pathways - Wnt and Shh - in a welldefined cohort of stem cells.

Using conditional alleles that allow activation of Shh and Wnt signaling, we found that activation of either signaling pathway in receiving cells in a tonic manner has a strong effect to increase the overall rate of neuron production in the dentate. Importantly, recent studies have clearly shown that Wnt signaling is not physiologically ever likely to be seen as a tonic phenomenon, but is rather bi- phasic in the dentate [34]. It would be valuable in future studies to identify methods allowing the biphasic activation of Wnt signaling in a more physiologic manner, as this would also likely avoid the problems with dendritic maturation and neuronal loss which we have seen in our study. However, what was quite clear is that activation of Wnt signaling in a tonic manner leads to a distinct pattern of neurogenesis compared to controls or activation of Shh signaling. Tonic Wnt signaling drives neurogenesis through an intermediate stage by increasing the pool of transient amplifying neurogenic precursors. The implication of this result is that the presence of both types of morphogenic ligands available to the dentate niche - Shh and Wnt - are necessary to help shape the type of neurogenic output seen from the dentate. This is quite intriguing in light of the recent work published by Dranovsky and colleagues [31] showing that neurogenesis in the dentate normally proceeds using a mixture of neurogenic modes, either including transient amplifying precursors or lacking them. Furthermore, they showed that environmental enrichment or stress led to changes in the relative balance of these two types of neurogenic output. This finding potentially links our results showing a disparity in the effects of Shh versus Wnt signaling activation to these different physiologic influences that shape the cellular makeup of the dentate.

It seems that activation of Wnt signaling in the expanding DG may have been the critical signaling development needed to meet the requirement to produce many neurons via a more rapid mode through the expansion of transient amplifying progenitors, while Shh signaling supports a baseline level of direct neurogenesis. It is interesting to consider the potential consequences of these different signaling pathways for the maintenance of longlived neural stem cells in the dentate. Recent studies from two labs using different methodologies have revealed that the dentate may be composed of several distinct types of neural stem cells - some competent to undergo long-term continued production of neurons over time via asymmetric divisions [35] versus others that seem to exhaust themselves in the production of new neurons [32]. The relationship between these different types of neurogenic divisions remains somewhat unclear and it is possible that technical differences in the approaches used to perform these studies [36] might be responsible for some of the differences. However, it is also possible that the different methods used in these studies led to distinct pools of neural stem cells being studied, and perhaps future studies may reveal that priming of neurogenesis by Shh versus Wnt may have distinct consequences for the long-term persistence and quiescence of SGZ stem cells. 
We found that Wnt activation in the RGC induces Cxcr4 gene expression in the dentate lineage and that Cxcr4 is a direct target of Lef1. Presumably, Cxcr4 is then responsive to Cxcl12 produced by the dentate granule cells themselves $[25,29,37]$. Our study also shows that conditional deletion of Cxcr4 in the postnatal dentate using the Gli1-CreERt2 driver line compromises the ability of dentate stem cells to generate the normal numbers of Tbr2+ transient amplifying precursors and that constitutively active Cxcr4 induces the production of IPCs from embryonic RG progenitors. This provides at least one insight into the mechanism whereby Wnt activation leads to the increased production of transient amplifying cells during neurogenesis. The fact that Cxcr4 is not absolutely required to allow dentate neurogenesis explains why previous studies failed to show a major neurogenic phenotype in the postanatal dentate other than the defects in organization seen in the embryonic dentate $[20,29,38]$. We believe that the roles of Cxcl12Cxcr4 signaling in the migration and organization of the dentate prenatally are important. But, to the extent that some cells are still able to reach the dentate, postnatal neurogenesis is able to be established and continues because of the multiple modes of neurogenesis that are possible in the dentate. However, we predict that conditional Cxcr4 mutant mice would respond abnormally to a variety of stimuli that are known to change the mode of dentate neurogenesis - e.g., environmental enrichment and stress [31].

Our studies revealing the roles of Shh and Wnt signaling in regulating two different modes of DG stem cell proliferation as well as the role of Cxcl12 signaling downstream of Wnt signaling may be important for understanding the role of these pathways in conditions such as depression and schizophrenia where alterations in neurogenesis in the dentate have been seen. Chronic stress represses Wnt signaling of the DG through expression of a Wnt inhibitor, Dickkopf-1, in the DG [39]. Based on our studies we would predict that this would compromise the ability of the dentate to continue transient precursor-mediated neurogenesis. Interestingly, we also now know that mutations in genes associated with schizophrenia and mental retardation can have important consequences for Wnt signaling [40, 41]. There are also some indications that changes in Wnt signaling in the dentate may be associated with declines in dentate neurogenesis associated with neurodegenerative diseases [42] and that restoring Wnt signaling might ameliorate these effects in experimental models [43-45]. Thus, we propose a model that a baseline ongoing mode of neurogenesis in the dentate is mostly dependent on continued Shh signaling but that the addition of Wnt activation drives the expansion of the pool of neurogenic precursors by supporting neurogenesis via transient amplifying precursors. This new framework may provide important clues for the expected roles of Shh and Wnt agonists in allowing the restoration or augmentation of dentate neurogenesis in a variety of disease states.

\section{Acknowledgements}

We thank Trung Huynh for technical assistance and Kurt Thorn and the Nikon Imaging Center at UCSF for use of the confocal microscope. This work was supported by R01 MH077694. S.J.P. is also supported by funds from the family of Glenn W. Johnson, Jr. The authors would also like to thank Dr. Alexandra Joyner for the use of the Gli1-CreERT2 mouse line.

\section{References}

$>_{1}$ Lie DC, Colamarino SA, Song HJ, Desire L, Mira H, Consiglio A, Lein ES, Jessberger S, Lansford H, Dearie AR, Gage FH: Wnt signalling regulates adult hippocampal neurogenesis. Nature 2005;437:1370-1375.

-2 Machold R, Hayashi S, Rutlin M, Muzumdar MD, Nery S, Corbin JG, Gritli-Linde A, Dellovade T, Porter JA, Rubin LL, Dudek H, McMahon AP, Fishell G: Sonic hedgehog is required for progenitor cell maintenance in telencephalic stem cell niches. Neuron 2003; 39:937-950.

3 Ahn S, Joyner AL: In vivo analysis of quiescent adult neural stem cells responding to sonic hedgehog. Nature 2005;437:894-897.
-4 Breunig JJ, Sarkisian MR, Arellano JI, Morozov YM, Ayoub AE, Sojitra S, Wang B, Flavell RA, Rakic P, Town T: Primary cilia regulate hippocampal neurogenesis by mediating sonic hedgehog signaling. Proc Natl Acad Sci USA 2008; 105:13127-13132.

5 Favaro R, Valotta M, Ferri AL, Latorre E, Mariani J, Giachino C, Lancini C, Tosetti V, Ottolenghi S, Taylor V, Nicolis SK: Hippocampal development and neural stem cell maintenance require Sox2-dependent regulation of Shh. Nat Neurosci 2009;12:12481256.

6 Han YG, Spassky N, Romaguera-Ros M, Garcia-Verdugo JM, Aguilar A, Schneider-
Maunoury S, Alvarez-Buylla A: Hedgehog signaling and primary cilia are required for the formation of adult neural stem cells. Nat Neurosci 2008;11:277-284.

7 Galceran J, Miyashita-Lin EM, Devaney E, Rubenstein JL, Grosschedl R: Hippocampus development and generation of dentate gyrus granule cells is regulated by LEF1. Development 2000;127:469-482.

8 Kuwabara T, Hsieh J, Muotri A, Yeo G, Warashina M, Lie DC, Moore L, Nakashima K, Asashima M, Gage FH: Wnt-mediated activation of NeuroD1 and retro-elements during adult neurogenesis. Nat Neurosci 2009;12: 1097-1105. 
-9 Zhou CJ, Zhao C, Pleasure SJ: Wnt signaling mutants have decreased dentate granule cell production and radial glial scaffolding abnormalities. J Neurosci 2004;24:121-126.

10 Bayer SA: Development of the hippocampal region in the rat. 2. Morphogenesis during embryonic and early postnatal life. J Comp Neurol 1980;190:115-134.

11 Li G, Pleasure SJ: Morphogenesis of the dentate gyrus: what we are learning from mouse mutants. Dev Neurosci 2005;27:93-99.

-12 Schlessinger AR, Cowan WM, Gottlieb DI: An autoradiographic study of the time of origin and the pattern of granule cell migration in the dentate gyrus of the rat. J Comp Neurol 1975;159:149-175.

13 Lee SM, Tole S, Grove E, McMahon AP: A local Wnt-3a signal is required for development of the mammalian hippocampus. Development 2000;127:457-467.

-14 Jessberger S, Clark RE, Broadbent NJ, Clemenson GD Jr, Consiglio A, Lie DC, Squire LR, Gage FH: Dentate gyrus-specific knockdown of adult neurogenesis impairs spatial and object recognition memory in adult rats. Learn Mem 2009;16:147-154.

-15 Zhuo L, Theis M, Alvarez-Maya I, Brenner M, Willecke K, Messing A: hGFAP-cre transgenic mice for manipulation of glial and neuronal function in vivo. Genesis 2001;31:8594.

16 Nie Y, Waite J, Brewer F, Sunshine MJ, Littman DR, Zou YR: The role of CXCR4 in maintaining peripheral $\mathrm{B}$ cell compartments and humoral immunity. J Exp Med 2004; 200:1145-1156.

17 Harada N, Tamai Y, Ishikawa T, Sauer B, Takaku K, Oshima M, Taketo MM: Intestinal polyposis in mice with a dominant stable mutation of the beta-catenin gene. EMBO J 1999;18:5931-5942.

- 18 Jeong J, Mao J, Tenzen T, Kottmann AH, McMahon AP: Hedgehog signaling in the neural crest cells regulates the patterning and growth of facial primordia. Genes Dev 2004; 18:937-951.

19 Choe Y, Siegenthaler JA, Pleasure SJ: A cascade of morphogenic signaling initiated by the meninges controls corpus callosum formation. Neuron 2012;73:698-712.

20 Bagri A, Gurney T, He X, Zou YR, Littman $\mathrm{DR}$, Tessier-Lavigne M, Pleasure SJ: The chemokine SDF1 regulates migration of dentate granule cells. Development 2002;129:42494260.

-21 Bamji SX, Shimazu K, Kimes N, Huelsken J, Birchmeier W, Lu B, Reichardt LF: Role of beta-catenin in synaptic vesicle localization and presynaptic assembly. Neuron 2003;40: 719-731.

-22 Yu X, Malenka RC: Beta-catenin is critical for dendritic morphogenesis. Nat Neurosci 2003;6:1169-1177.
23 Hodge RD, Kowalczyk TD, Wolf SA, Encinas JM, Rippey C, Enikolopov G, Kempermann G, Hevner RF: Intermediate progenitors in adult hippocampal neurogenesis: Tbr2 expression and coordinate regulation of neuronal output. J Neurosci 2008;28:3707-3717.

24 Jho EH, Zhang T, Domon C, Joo CK, Freund JN, Costantini F: Wnt/beta-catenin/Tcf signaling induces the transcription of Axin2, a negative regulator of the signaling pathway. Mol Cell Biol 2002;22:1172-1183.

25 Berger O, Li G, Han SM, Paredes M, Pleasure SJ: Expression of SDF-1 and CXCR4 during reorganization of the postnatal dentate gyrus. Dev Neurosci 2007;29:48-58.

26 Bhattacharyya BJ, Banisadr G, Jung H, Ren D, Cronshaw DG, Zou Y, Miller RJ: The chemokine stromal cell-derived factor-1 regulates gabaergic inputs to neural progenitors in the postnatal dentate gyrus. J Neurosci 2008;28:6720-6730.

27 Munji RN, Choe Y, Li G, Siegenthaler JA, Pleasure SJ: Wnt signaling regulates neuronal differentiation of cortical intermediate progenitors. J Neurosci 2011;31:1676-1687.

28 Mutch CA, Schulte JD, Olson E, Chenn A: Beta-catenin signaling negatively regulates intermediate progenitor population numbers in the developing cortex. PLoS One 2010;5:e12376.

-29 Li G, Kataoka H, Coughlin SR, Pleasure SJ: Identification of a transient subpial neurogenic zone in the developing dentate gyrus and its regulation by $\mathrm{Cxcl} 12$ and reelin signaling. Development 2009;136:327-335.

30 Zhao C, Deng W, Gage FH: Mechanisms and functional implications of adult neurogenesis. Cell 2008;132:645-660.

31 Dranovsky A, Picchini AM, Moadel T, Sisti AC, Yamada A, Kimura S, Leonardo ED, Hen R: Experience dictates stem cell fate in the adult hippocampus. Neuron 2011;70:908923.

32 Encinas JM, Michurina TV, Peunova N, Park JH, Tordo J, Peterson DA, Fishell G, Koulakov A, Enikolopov G: Division-coupled astrocytic differentiation and age-related depletion of neural stem cells in the adult hippocampus. Cell Stem Cell 2011;8:566-579.

-33 Lagace DC, Whitman MC, Noonan MA, Ables JL, DeCarolis NA, Arguello AA, Donovan MH, Fischer SJ, Farnbauch LA, Beech RD, DiLeone RJ, Greer CA, Mandyam CD, Eisch AJ: Dynamic contribution of nestinexpressing stem cells to adult neurogenesis. J Neurosci 2007;27:12623-12629.

34 Garbe DS, Ring RH: Investigating tonic Wnt signaling throughout the adult CNS and in the hippocampal neurogenic niche of BatGal and Ins-TopGal mice. Cell Mol Neurobiol 2012.

35 Bonaguidi MA, Wheeler MA, Shapiro JS, Stadel RP, Sun GJ, Ming GL, Song H: In vivo clonal analysis reveals self-renewing and multipotent adult neural stem cell characteristics. Cell 2011;145:1142-1155.
36 Kempermann G: The pessimist's and optimist's views of adult neurogenesis. Cell 2011; 145:1009-1011.

37 Kolodziej A, Schulz S, Guyon A, Wu DF, Pfeiffer M, Odemis V, Hollt V, Stumm R: Tonic activation of CXC chemokine receptor 4 in immature granule cells supports neurogenesis in the adult dentate gyrus. J Neurosci 2008;28:4488-4500.

38 Lu M, Grove EA, Miller RJ: Abnormal development of the hippocampal dentate gyrus in mice lacking the CXCR4 chemokine receptor. Proc Natl Acad Sci USA 2002;99:70907095.

39 Matrisciano F, Busceti CL, Bucci D, Orlando R, Caruso A, Molinaro G, Cappuccio I, Riozzi B, Gradini R, Motolese M, Caraci F, Copani A, Scaccianoce S, Melchiorri D, Bruno V, Battaglia G, Nicoletti F: Induction of the Wnt antagonist Dickkopf-1 is involved in stress-induced hippocampal damage. PLoS One 2011;6:e16447.

40 Luo Y, Shan G, Guo W, Smrt RD, Johnson EB, Li X, Pfeiffer RL, Szulwach KE, Duan R, Barkho BZ, Li W, Liu C, Jin P, Zhao X: Fragile $\mathrm{x}$ mental retardation protein regulates proliferation and differentiation of adult neural stem/progenitor cells. PLoS Genet 2010;6:e1000898.

41 Mao Y, Ge X, Frank CL, Madison JM, Koehler AN, Doud MK, Tassa C, Berry EM, Soda T, Singh KK, Biechele T, Petryshen TL, Moon RT, Haggarty SJ, Tsai LH: Disrupted in schizophrenia 1 regulates neuronal progenitor proliferation via modulation of GSK3beta/beta-catenin signaling. Cell 2009;136: 1017-1031.

-42 Fuster-Matanzo A, Llorens-Martin M, de Barreda EG, Avila J, Hernandez F: Different susceptibility to neurodegeneration of dorsal and ventral hippocampal dentate gyrus: a study with transgenic mice overexpressing GSK3beta. PLoS One 2011;6:e27262.

43 De Ferrari GV, Chacon MA, Barria MI, Garrido JL, Godoy JA, Olivares G, Reyes AE, Alvarez A, Bronfman M, Inestrosa NC: Activation of Wnt signaling rescues neurodegeneration and behavioral impairments induced by beta-amyloid fibrils. Mol Psychiatry 2003; 8:195-208

44 Rockenstein E, Torrance M, Adame A, Mante M, Bar-on P, Rose JB, Crews L, Masliah E: Neuroprotective effects of regulators of the glycogen synthase kinase-3beta signaling pathway in a transgenic model of Alzheimer's disease are associated with reduced amyloid precursor protein phosphorylation. J Neurosci 2007;27:1981-1991.

45 Toledo EM, Inestrosa NC: Activation of Wnt signaling by lithium and rosiglitazone reduced spatial memory impairment and neurodegeneration in brains of an APPswe/ PSEN1DeltaE9 mouse model of Alzheimer's disease. Mol Psychiatry 2010;15:272-285, 228. 